\title{
A Feedback Control Model for Freeways Based on Monitoring System
}

\author{
Xingju Wang ${ }^{1,2^{*}}$, Yuehong Liu ${ }^{1}$, Yang Zhou ${ }^{1}$ and Xiaoming $\mathrm{Xi}^{3}$ \\ ${ }^{1}$ School of Traffic and Transportation \\ Shijiazhuang Tiedao University \\ Hebei, Shijiazhuang, 050043, China \\ ${ }^{2}$ Key Laboratory of Traffic Safety and Control of Hebei Province \\ Hebei, Shijiazhuang, 050043, China \\ ${ }^{3}$ China Railway Shanghai Design Institute Group \\ Tianjin, 300000, China \\ wangxingju@stdu.edu.cn
}

\begin{abstract}
In recent years, the freeway monitoring systemis widely used in China. Based on the data from these monitoring systems, real time control is able to be employed on the freeway under consideration of such uncertain factors as haze, agglomerate flog, accident. A mainline feedback control model is proposed in terms of monitoring system data and feedback control theory. A freeway in Hebei is taken as a case study to validate the proposed feedback control model using traffic flow simulation of weekday off-peak period, weekday peak period, holiday off-peak period, holiday peak period, severe weather off-peak period and severe weather peak period. Results show traffic flow is smooth and local congestion is alleviated in weekday period cases with less volume. However, travel time increases in weekday period cases. On the other hand, in the other cases with more volume, not only traffic flow is smooth, but also travel time decreases. On top of that, travel time falls by about $9 \%$ in severe weather peak period case.
\end{abstract}

Keywords: Feedback control, monitoring system, jam, ramp metering, traffic flow simulation, control density

\section{Introduction}

Freeways play a key role in transportation in China. Freeway network has been rapidly growing in China during the decades. By the end of 2014, China has 111950 kilometers $(\mathrm{km})$ freeway operation mileage, making it the busiest freeway transportation system in the world. With the rapid evolution of economy and large number of vehicles, the carbased transportation has led to the daily occurrence and recurrent congestions in China. Recurrent jams reduce substantially the available infrastructure capacity, cause delays and create traffic safety risks. In addition, jams also increase the air pollution, which has caused widespread environmental and healthy problems. Freeway monitoring and control systems are essential to the efficient operation of freeways, particularly when volumes are high. Freeway monitoring systems are widely used and extended to freeway management and operation in China.

Recently, researchers showed great interest in freeway traffic flow control approaches. R. Boel and L. Mihaylova [1] presented a stochastic model of freeway traffic at a time scale. W. Brilon and J. Lohoff [2] provided several models to discuss each state of traffic flow by one function. H. B. Celikoglu [3] focused on the travel time performance function throughout fundamental diagram relation under congested and un-congested traffic patterns. Some neural network models were used to forecast traffic flow $[4,5]$. Some 
novel models were extended to predict travel time [6-8]. Several important methodological developments and applications have been undertaken on the traffic safety analysis on freeways using traffic flow models [9, 10]. Published papers provided a comprehensive methodological approach to study ramp metering [11, 12]. Various models have been constructed to show the control approaches [13-15]. Also, recent studies provide insights related to analyze real data, which was detected on freeways [16, 17]. The aim of this study is to propose a feedback control model for freeways under unfavorable conditions in terms of real dynamic data, which is able to be detected on the whole freeway.

\section{Methodology}

Freeway monitoring systems, which provide the traffic flow parameters such as flow rate, density and mean speed, are widely used in China. This paper proposes a feedback control model for freeways based on a monitoring system. Assume a freeway is composed of $N$ basic sections, whose traffic flow parameters can be given by the monitoring system as shown in Figure 1. The basic control model for freeways is denoted by

\begin{tabular}{|l|l|l|l|l|l|l|l|}
\hline 1 & 2 & $\ldots$ & $\mathrm{i}-1$ & $\mathrm{i}$ & $\mathrm{i}+1$ & $\ldots$ & $\mathrm{N}$ \\
\hline
\end{tabular}

Figure 1. View of Basic Sections on the Freeway

$$
\hat{\rho}_{i}(k+1)=\hat{\rho}_{i}(k)+f\left(\alpha_{i}, \beta_{i}\right)\left|\hat{\rho}_{i}(k)-\rho_{i}(k)\right|
$$

where

$\hat{\rho}_{i}(k+1)$ is the control traffic density of period $k+1$ in section $i$;

$\hat{\rho}_{i}(k)$ is the control traffic density of period $k$ in section $i$;

$\rho_{i}(k)$ is the actual traffic density of period $k$ in section $i$;

$\alpha_{i}$ is the endogenous influence index in section $i\left(0<\alpha_{i}<1\right)$;

$\beta_{i}$ is the exogenous influence index in section $i$, on which other sections impact $\left(0<\beta_{i}<1\right)$;

$f\left(\alpha_{i}, \beta_{i}\right)$ is the correlation coefficient function for $\alpha_{i}$ and $\beta_{i}$.

The constraints are as following

$$
\begin{aligned}
& -\eta \leq \hat{\rho}_{i}(k)-\hat{\rho}_{i+1}(k) \leq \eta \\
& -\lambda \leq \hat{\rho}_{i}(k)-\hat{\rho}_{i}(k+1) \leq \lambda \\
& \theta \leq \hat{\rho}_{i}(k) \leq \omega
\end{aligned}
$$

where

$\eta$ is an index with $\eta>0$, presenting the control density difference between adjacent sections;

$\lambda$ is an index with $\lambda>0$, presenting the control density difference of the same section between adjacent period;

$\theta$ is the minimum control density with $\theta>0$;

$\omega$ is the maximum control density with $\omega>0$. 
A traffic bottleneck is a localized disruption of vehicular traffic on a street, road or freeway. As opposed to a traffic jam, a bottleneck is a result of a specific physical condition, often the design of the road, badly timed traffic lights or sharp curves. This paper divided freeway sections into non-ramp bottleneck sections, ramp bottleneck sections and non-bottleneck sections. The basic model is rewritten by control models for non-ramp bottleneck sections, ramp bottleneck sections and non-bottleneck sections, respectively.

A non-ramp bottleneck section $n$ exits and $n \in[1, N]$. Assume that the bottleneck section is independent, respectively. Then, we have

$$
f\left(\alpha_{n}, \beta_{n}\right)=g\left(\alpha_{n}\right)=H\left(\hat{\rho}_{n}(k), \rho_{n}(k)\right) \cdot h
$$

where

$g\left(\alpha_{n}\right)$ is the endogenous influence function of bottleneck section $n$;

$h$ is an adjustment coefficient, presenting the ratio of adjusting density $(0<h<1)$;

$H\left(\hat{\rho}_{n}(k), \rho_{n}(k)\right)$ is a direction judgment coefficient and is defined by

$$
H\left(\hat{\rho}_{n}(k), \rho_{n}(k)\right)=\frac{\hat{\rho}_{n}(k)-\rho_{n}(k)}{\left|\hat{\rho}_{n}(k)-\rho_{n}(k)\right|}
$$

The control model of the ramp bottleneck sections, which comes from Equation (1), is rewritten by

$$
\hat{\rho}_{n}(k+1)=\hat{\rho}_{n}(k)+H\left(\hat{\rho}_{n}(k), \rho_{n}(k)\right) \cdot h \cdot\left|\hat{\rho}_{n}(k)-\rho_{n}(k)\right|
$$

Ramp metering is essential to the efficient operation of freeways, particularly when volumes are high. According to Papageorgiou and others, ramp metering is divided roughly into the reacted type and the preceded type [18]. DC (Demand-Capacity), OCC (Occupancy), ALNEA[19] are among the well-known local response type ramp metering [20]. In this paper, the ALNEA model is applied to conduct ramp metering. A ramp bottleneck section $m$ exits and $m \in[1, N]$ as shown in Figure 2. The control model of ramp bottleneck sections is presented by

$r_{m}(k+1)=r_{m}(k)-K_{R}\left[\rho_{m}(k)-\hat{\rho}_{m}(k)\right]$

where

$r_{m}(k+1)$ is the metering rate of period $k+1$ in ramp bottleneck section $m$;

$r_{m}(k)$ is the metering rate of period $k$ in ramp bottleneck section $m$;

$K_{R}$ is a positive constant.

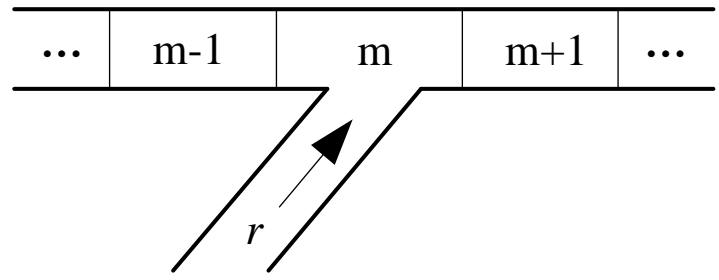

Figure 2. View of the Ramp Bottleneck Section

Also assume that each bottleneck section is independent, indicating two bottleneck section can not impact on the same non-bottleneck sections. A non-bottleneck section $j$ and bottleneck section $n$ exit and $j, n \in[1, N]$ as shown in Figure 3. Define that $d$ is a positive constant, presenting the influence scope of bottleneck section $n$. 


\begin{tabular}{l|c|c|c|cc}
\hline $\mathrm{n}-\mathrm{d} \cdots$ & $\mathrm{n}-1$ & $\mathrm{n}$ & $\mathrm{n}+1$ & $\cdots$ & $\mathrm{n}+\mathrm{d}$ \\
\hline
\end{tabular}

Figure 3. View of Non-Ramp Bottleneck Sections

The control model of non-bottleneck sections is discussed as following

When $j \in[n-d, n+d]$ and $j \neq n$, we have

$$
\begin{aligned}
& g\left(\alpha_{j}\right)=H\left(\hat{\rho}_{j}(k), \rho_{j}(k)\right) \cdot h \\
& t_{n}\left(\beta_{j}\right)=H\left(\hat{\rho}_{j}(k), \rho_{j}(k)\right) \cdot \frac{|j-n|}{2 d}
\end{aligned}
$$

where $t_{n}\left(\beta_{j}\right)$ is the exogenous influence function in section $j$, on which section $n$ impact.

With $g\left(\alpha_{j}\right)>0$ and $t_{n}\left(\beta_{j}\right)>0$, we have $\frac{g\left(\alpha_{j}\right)}{t_{n}\left(\beta_{j}\right)}>0$. The correlation coefficient function $f\left(\alpha_{j}, \beta_{j}\right)$ is expressed by

$$
f\left(\alpha_{j}, \beta_{j}\right)=\min \left(g\left(\alpha_{j}\right), t_{n}\left(\beta_{j}\right)\right)
$$

With $g\left(\alpha_{j}\right)>0$ and $t_{n}\left(\beta_{j}\right)<0$, we have $\frac{g\left(\alpha_{j}\right)}{t_{n}\left(\beta_{j}\right)}<0 . f\left(\alpha_{j}, \beta_{j}\right)$ is expressed by

$$
f\left(\alpha_{j}, \beta_{j}\right)=t_{n}\left(\beta_{j}\right)
$$

With $g\left(\alpha_{j}\right)<0$ and $t_{n}\left(\beta_{j}\right)>0$, we have $\frac{g\left(\alpha_{j}\right)}{t_{n}\left(\beta_{j}\right)}<0, f\left(\alpha_{j}, \beta_{j}\right)$ is expressed by

$$
f\left(\alpha_{j}, \beta_{j}\right)=g\left(\alpha_{j}\right)
$$

With $g\left(\alpha_{j}\right)<0$ and $t_{n}\left(\beta_{j}\right)<0$, we have $\frac{g\left(\alpha_{j}\right)}{t_{n}\left(\beta_{j}\right)}>0 . f\left(\alpha_{j}, \beta_{j}\right)$ is expressed by

$$
f\left(\alpha_{j}, \beta_{j}\right)=\min \left(g\left(\alpha_{j}\right), t_{n}\left(\beta_{j}\right)\right)
$$

(2) When $j \notin[n-d, n+d]$, we obtain

$$
f\left(\alpha_{j}, \beta_{j}\right)=g\left(\alpha_{j}\right)
$$

The control traffic density model of each section on the freeway has been proposed. The corresponding control speed can be obtained by the fitting density-speed curve. On the top of these traffic parameters, traffic control plan is conducted in terms of the monitoring system on the freeway. In Figure 4, the feedback control diagram is illustrated as following:

Step 1: The traffic density of each section is detected by the freeway monitoring system;

Step 2: Judge whether the section is the bottleneck; if not, then go to step 3. Otherwise go to step 4.

Step 3: Judge whether the section is impacted by the other bottleneck section. Then decide $f\left(\alpha_{j}, \beta_{j}\right)$ in light of the control model of non-bottleneck section. 
Step 4: Judge whether the section is the ramp section. If yes, then conduct ramp metering control and go to step 6. Otherwise decide the $f\left(\alpha_{j}, \beta_{j}\right)$ based on the control model of the non-ramp bottleneck section.

Step 5: On the top of $f\left(\alpha_{j}, \beta_{j}\right)$, the control traffic density $\hat{\rho}_{i}(k+1)$ is obtained according to the basic feedback control model.

Step 6: The speed control plan is made in terms of the control density of each section on the freeway.

Step 7: Judge whether the control is over. If yes, stop the control. Otherwise update the control cycle and return to step 1.

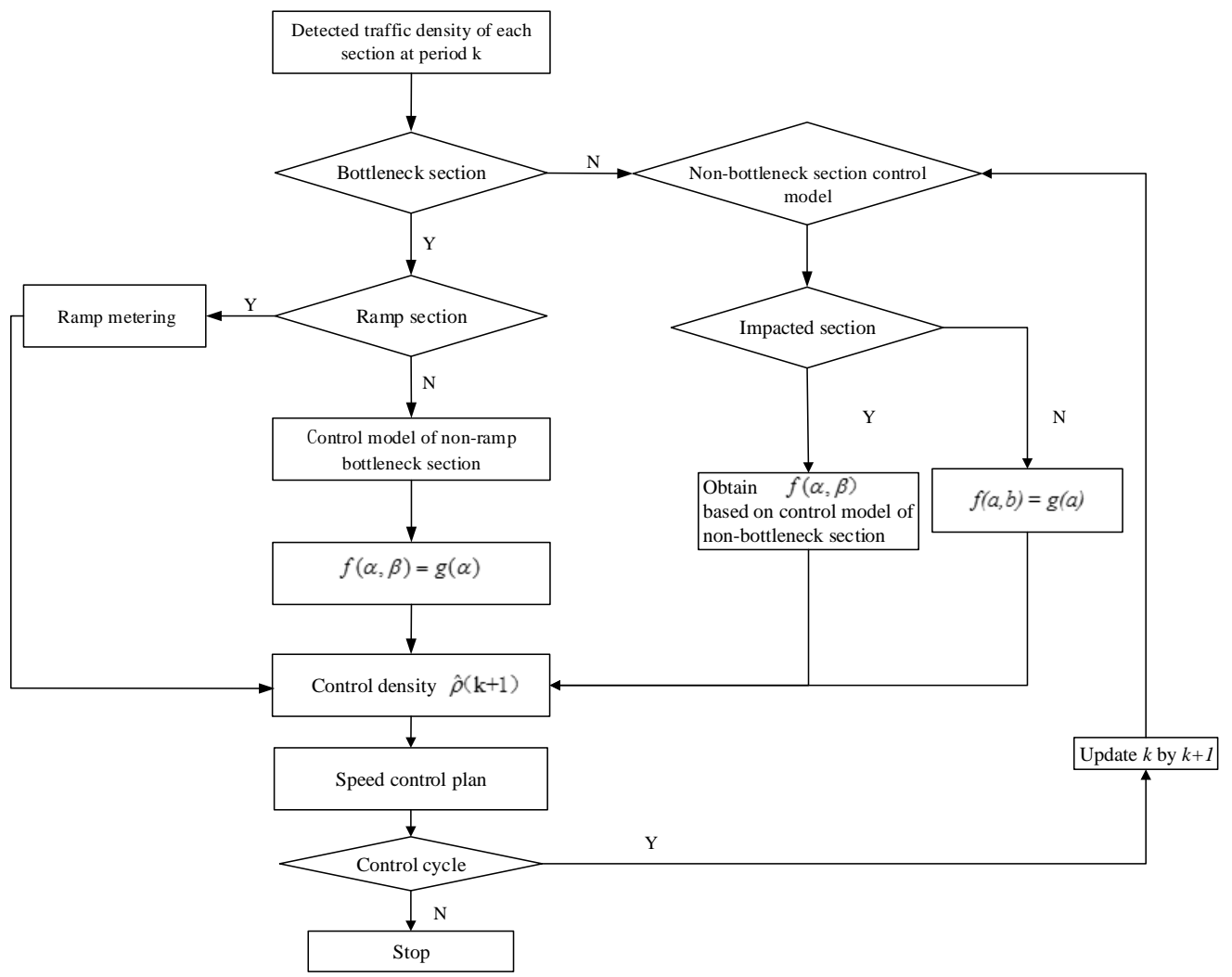

Figure 4. Diagram of Feedback Control Model

\section{Case Study}

A freeway in Hebei was taken as the case study to validate the proposed feedback control model. The freeway is 47 kilometers $(\mathrm{km})$ and installed a traffic monitoring information system composed of cameras, variable message sign, detector, radio, horn, etc. on the whole freeway. The detected data was collected from the whole traffic monitoring information system. The dealt data was displayed in the monitoring center. The outer equipment distribution and sections were shown in Figure 5. The freeway starts at a toll-gate and ends at a toll-gate. There are 28 nodes and 27 sections composed of an on-ramp bottleneck section, 3 non-ramp bottleneck sections and 23 non-bottleneck sections. The traffic parameters of each section are detected by the corresponding professional camera. Moreover, an agglomerate fog district and a long bridge are paid great attention to. The distance between two cameras is $2 \mathrm{~km}$ for non-bottleneck sections. On the other hand, the distance for the bottleneck sections decreases to $1 \mathrm{~km}$. 

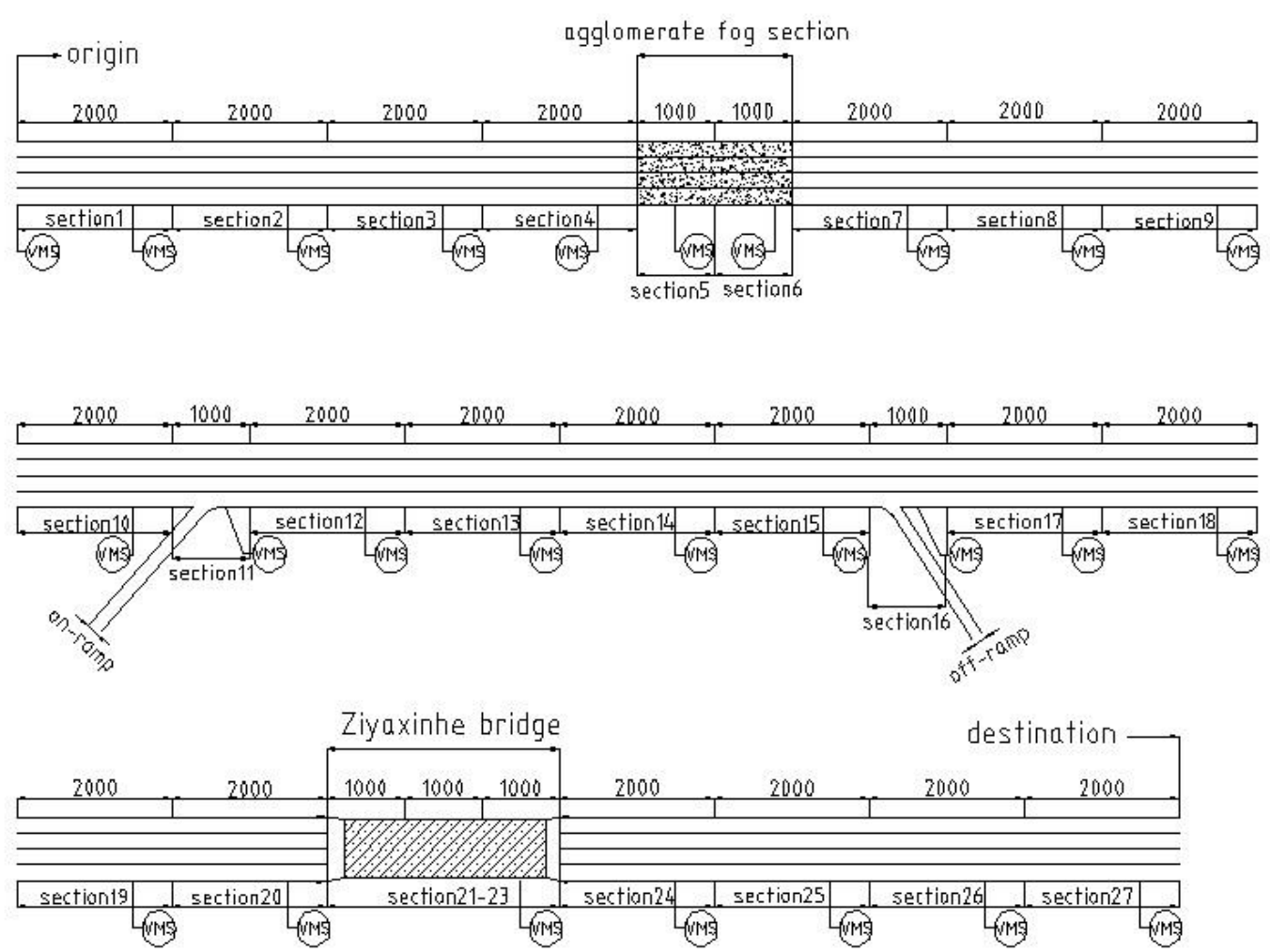

\section{Figure 5. View of Outer Equipment and Sections on the freeway of Hebei Province in China}

The proposed feedback control models for the whole freeway were applied to control traffic flow. Microscopic traffic flow simulation was used to validate these proposed models. The parameters for these proposed models were indicated in Table 1. There were 6 cases with weekday off-peak period, weekday peak period, holiday off-peak period, holiday peak period, severe weather off-peak period under stress of weather and severe weather peak period under stress of weather. The traffic parameters for the general period were collected between 11:00 and 16:00. The traffic parameters for the peak period were obtained from 9:00 to 11:00. The traffic parameters for holidays were given by the National Day holiday between October 1 and 7 from 2008 to 2011. These traffic parameters were presented in Table 2. The parameters for vehicle category were shown in Table 3 in terms of the detected real data using the traffic monitoring information system. The vehicle conversion coefficient is set at Table 4 . The control density of each section was addressed using these models. On the top of control density, speed limit plan of each section was made to smooth the traffic flow on the freeway. According to the real detected speed and density of each section on the freeway, the relationship or fitting curve between the speed and density of sections was represented in Figure 6. The red curve described the relationship between the real detected speed and density. On the other hand, the black line indicated the fitting the curve. $R$ is the distance between the real curve and fitting curve and $R \in[0,1] . R^{2}$ is the goodness of fit. In this case study, the $R^{2}$ arrived at 0.9983 . According to this curve, the control speed plan was made based on the obtained control density of each section. Furthermore, the corresponding control speed of each section was represented on the corresponding information board. The drivers on the freeway adjust their speed in light of proposed information in the corresponding boards. 
Table 1. Parameters for the Feedback Control Model

\begin{tabular}{cccccccc}
\hline Parameter & $k / \mathbf{s}$ & $\begin{array}{c}\eta / \\
\left(\mathbf{p c u} \cdot \mathbf{k m}^{-\mathbf{1}}\right)\end{array}$ & $\begin{array}{c}\lambda / \\
\left(\mathbf{p c u} \cdot \mathbf{k m}^{-\mathbf{1}}\right)\end{array}$ & $\begin{array}{c}\theta / \\
\left(\mathbf{p c u} \cdot \mathbf{k m}^{-\mathbf{1}}\right)\end{array}$ & $\begin{array}{c}\omega / \\
\left(\mathbf{p c u} \cdot \mathbf{k m}^{-\mathbf{1}}\right)\end{array}$ & $\begin{array}{c}\mu / \\
\left(\mathbf{k m} \cdot \mathbf{h}^{-}\right.\end{array}$ & $\begin{array}{c}\xi / \\
\mathbf{1})\end{array}$ \\
\hline Value & 3600 & 20 & 30 & 10 & 100 & 30 & 120 \\
\hline
\end{tabular}

Table 2. Traffic Flow Parameters

\begin{tabular}{ccccccc}
\hline Case & $\mathbf{1}$ & $\mathbf{2}$ & $\mathbf{3}$ & $\mathbf{4}$ & $\mathbf{5}$ & $\mathbf{6}$ \\
\hline Mainline traffic volumes $(\mathbf{p c u} / \mathbf{h})$ & 1200 & 1500 & 1800 & 2000 & 2300 & 2500 \\
Ramp traffic volumes $(\mathbf{p c u} / \mathbf{h})$ & 300 & 300 & 600 & 900 & 600 & 900 \\
Total traffic volumes $(\mathbf{p c u} / \mathbf{h})$ & 1500 & 1800 & 2400 & 2900 & 2900 & 3400 \\
\hline
\end{tabular}

Table 3. Parameters for Vehicle Category

\begin{tabular}{ccc}
\hline category & desired speed $(\mathrm{km} / \mathrm{h})$ & proportion \\
\hline cars & 120 & 0.6 \\
trucks & 90 & 0.3 \\
buses & 70 & 0.1 \\
\hline
\end{tabular}

Table 4. Vehicle Conversion Coefficient

\begin{tabular}{ccc}
\hline category & conversion coefficient & condition \\
\hline small truck & 1.0 & workload $\leq 2 t$ \\
medium truck & 1.5 & $2 t<$ workload $\leq 7 t$ \\
heavy truck & 2.0 & $7 t<$ workload $\leq 14 t$ \\
heavy truck & 3.0 & workload $>14 t$ \\
small bus & 1.0 & rated seat $\leq 19$ seats \\
large bus & 1.5 & rated seat $>19$ seats \\
\hline
\end{tabular}

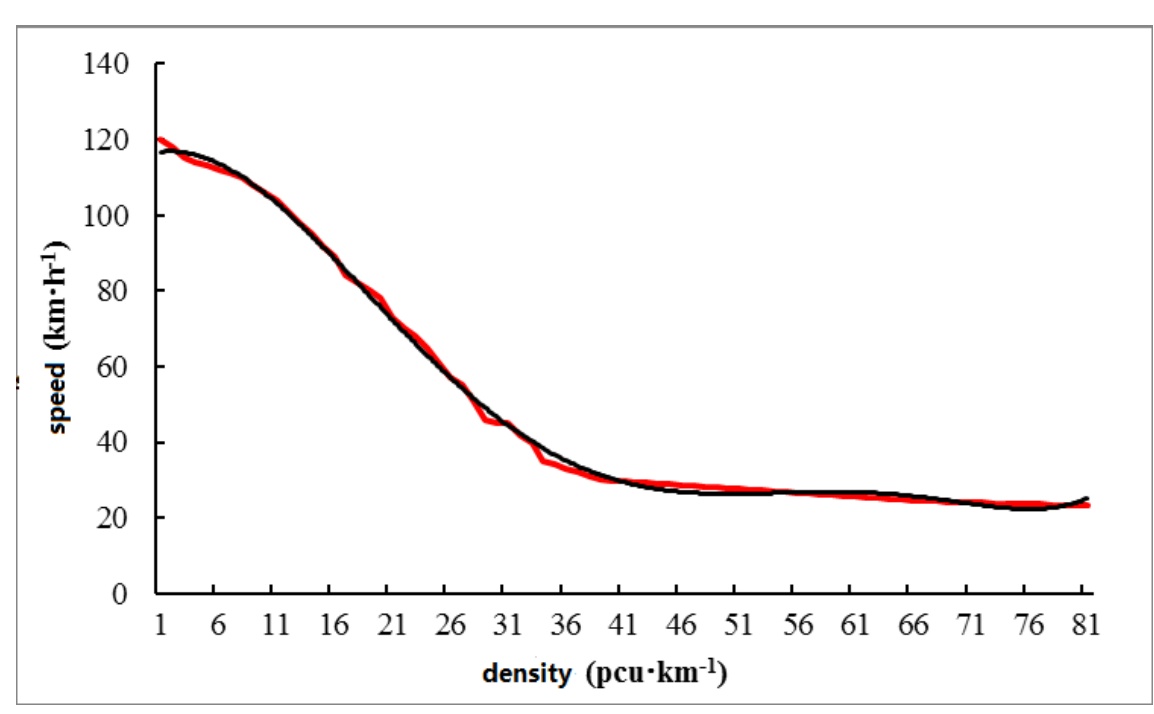

Figure 6. Fitting Curve between Speed and Density 


\section{Results and Discussion}

In this study, a freeway in Hebei was taken as case study to validate the proposed feedback control model using traffic flow simulation of weekday off-peak period, weekday peak period, holiday off-peak period, holiday peak period, severe weather offpeak period and severe weather peak period. Results of cases were indicated in Table 5 and Figure 7. Table 5 presented the control density of each section with control and without control. In Table 5, section 5, section 6, section 11, section 16 and section 21, section 22, and section 23 are bottleneck sections as indicated by the gray color. The effectiveness of control in case 5 was clear in terms of density of bottleneck sections. The traffic flow was smooth. On the other hand, since traffic volume was low in case 1 and case 2, the whole traffic flow control had no effect. Figure 7 represented the results of the proposed control model using the travel time. Travel time with control increased $1 \mathrm{~s}$ in case 1 for weekday off-peak period. The result showed that the whole control for the freeway had little effect on travel time. However, in case 2 for weekday peak period, the travel time with control increased $139 \mathrm{~s}$ compared to no control. On the other hand, travel time with control decreased $102 \mathrm{~s}, 210 \mathrm{~s}$, and $352 \mathrm{~s}$ in case 3 , case 4 and case 5 . The control effectiveness was obviously represented in cases of the high traffic flow.

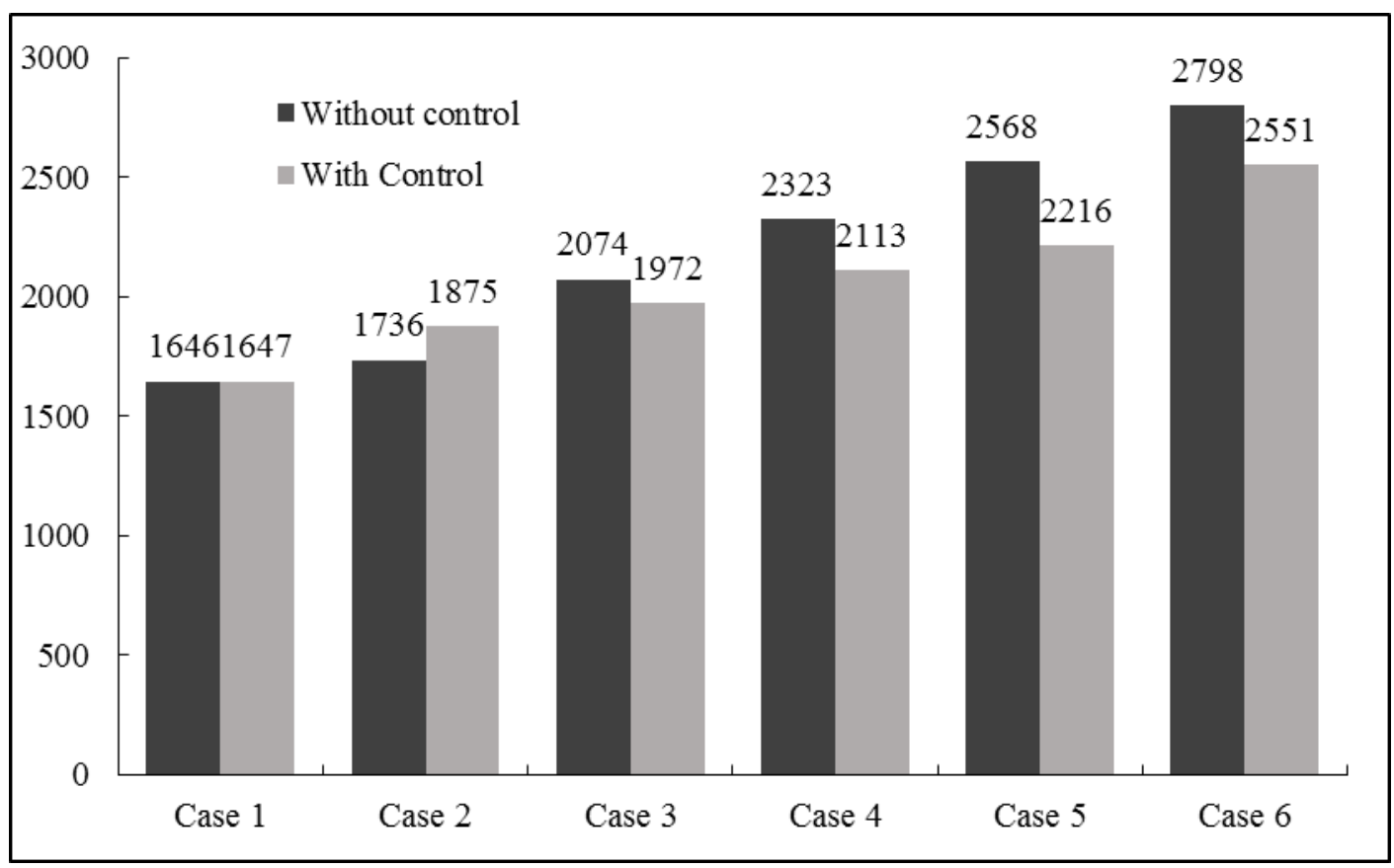

Figure 7. Average Travel Time with Control and without Control 
Table 5. Control Density of Each Section with Control and Without Control

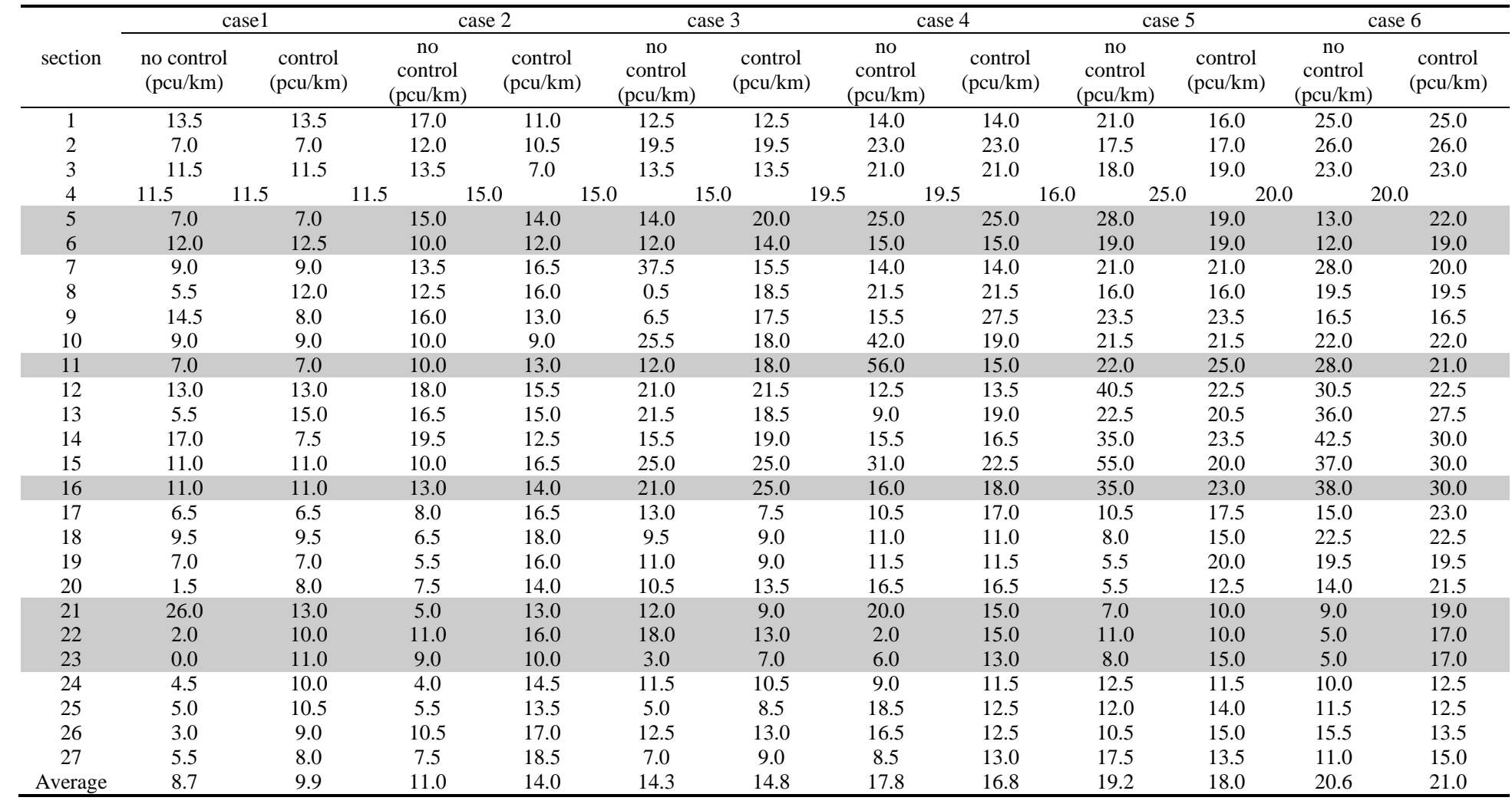

\section{Conclusion}

According to easing traffic congestion, the whole control for freeways had been conducted in China. The engineers and researchers in China focused on the corresponding control model for the whole freeway. This paper proposed feedback control models for freeways in light of traffic stream characters for bottleneck sections and non-bottleneck sections. The freeway in Hebei was taken as a case study to validate the proposed feedback control model using traffic flow simulation of weekday off-peak period, weekday peak period, holiday off-peak period, holiday peak period, severe weather offpeak period and severe weather peak period. The results showed the whole control had an obvious effect for the sections with the high traffic volume using the travel time and density. On the other hand, there was little effective for sections with the low traffic volume.

The current freeway control model mostly pays attention to the local control for ramp metering. However, the monitoring system for the whole freeway had been widely used in China. Though a comprehensive control model for the whole freeway based on the monitoring system has been proposed and discussed in this paper, additional research will be conducted to further improve the model and its applications in the future. One of such improvements is to extend the model to the vehicle infrastructure system. Another issue that deserves attention is how to control the traffic flow based on the detected big data. However, multiple model based on the monitoring system, vehicle infrastructure system, and big data is challenging and deserves research efforts in the future. 


\section{Acknowledgements}

This research was funded by the Natural Science Foundation of China (Grant No: 51008201), Natural Science Foundation of Hebei Province in China (Grant No: E2012210016 and E2014210152), Science Program of Hebei Province in China (Grant No: 13455408D and 13456236D), and the Scientific Research Foundation of Education Department of Hebei Province for Outstanding Young Teachers in University in China (Grant No. Y2012033 and ZD2014084). This research is further sponsored by the Outstanding Young Talent Foundation of Hebei Province in China, Talent Program of Hebei Province in China (Grant No. A201400212), the Outstanding Young Talent Foundation of Shijiazhuang Tiedao University in China and Hebei Social Science Research Center for Project Construction Management.

\section{References}

[1] R. Boel and L. Mihaylova, "A compositional stochastic model for real time freeway traffic simulation", Transportation Research Part B: Methodological, vol. 40, no. 5, (2006), pp. 319-334.

[2] W. Brilon and J. Lohoff, "Speed-flow Models for Freeways", Procedia - Social and Behavioral Sciences, vol. 16, (2011), pp. 26-36.

[3] H. B. Celikoglu, "Reconstructing freeway travel times with a simplified network flow model alternating the adopted fundamental diagram", European Journal of Operational Research, vol. 228, no. 2, (2013), pp. 457-466.

[4] H. Dia and G. Rose, "Development and evaluation of neural network freeway incident detection models using field data", Transportation Research Part C: Emerging Technologies, vol. 5, no. 5, (1997), pp. 313-331.

[5] A. Dharia and H. Adeli, "Neural network model for rapid forecasting of freeway link travel time", Engineering Applications of Artificial Intelligence, vol. 16, no. 7-8, (2003), pp. 607-613.

[6] X. Fei, C.-C. Lu and K. Liu, "A bayesian dynamic linear model approach for real-time short-term freeway travel time prediction", Transportation Research Part C: Emerging Technologies, vol. 19, no. 6, (2011), pp. 1306-1318.

[7] J. R. D. Frejo, A. Núñez, B. De Schutter and E. F. Camacho, "Hybrid model predictive control for freeway traffic using discrete speed limit signals", Transportation Research Part C: Emerging Technologies, vol. 46, (2014), pp. 309-325.

[8] Y. Qi and S. Ishak, "A Hidden Markov Model for short term prediction of traffic conditions on freeways", Transportation Research Part C: Emerging Technologies, vol. 43, Part 1, (2014), pp. 95-111.

[9] I. Ghosh, "Examination of the Factors Influencing the Clearance Time of Freeway Incidents", Journal of Transportation Systems Engineering and Information Technology, vol. 12, no. 3, (2012), pp. 75-89.

[10] T. F. Golob, W. Recker and Y. Pavlis, "Probabilistic models of freeway safety performance using traffic flow data as predictors", Safety Science, vol. 46, no. 9, (2008), pp. 1306-1333.

[11] G. Gomes and R. Horowitz, "Optimal freeway ramp metering using the asymmetric cell transmission model", Transportation Research Part C: Emerging Technologies, vol. 14, no. 4, (2006), pp. 244-262.

[12] A. Spiliopoulou, M. Kontorinaki, M. Papageorgiou and P. Kopelias, "Macroscopic traffic flow model validation at congested freeway off-ramp areas", Transportation Research Part C: Emerging Technologies, vol. 41, (2014), pp. 18-29.

[13] A. Kondyli, I. Soria, A. Duret and L. Elefteriadou, "Sensitivity analysis of CORSIM with respect to the process of freeway flow breakdown at bottleneck locations", Simulation Modelling Practice and Theory, vol. 22, (2012), pp. 197-206.

[14] I. Papamichail, A. Kotsialos, I. Margonis and M. Papageorgiou, "Coordinated ramp metering for freeway networks - A model-predictive hierarchical control approach", Transportation Research Part C: Emerging Technologies, vol. 18, no.3, (2010), pp. 311-331.

[15] A. Srivastava and N. Geroliminis, "Empirical observations of capacity drop in freeway merges with ramp control and integration in a first-order model", Transportation Research Part C: Emerging Technologies, vol. 30, (2013), pp. 161-177.

[16] P. Strömgren, "Analysis of the Weaknesses in the Present Freeway Capacity Models for Sweden", Procedia - Social and Behavioral Sciences, vol. 16, (2011), pp. 76-88.

[17] M. Hossain and Y. Muromachi, "A Bayesian network based framework for real-time crash prediction on the basic freeway segments of urban expressways", Accident Analysis \& Prevention, vol. 45, (2012), pp. 373-381.

[18] M. Papageorgiou and A. Kotsialos, "Freeway ramp metering: an overview", in Intelligent Transportation Systems, 2000. Proceedings. 2000 IEEE, 2000, vol. 3, no.4, (2002),pp. 228-239.

[19] M. Papageorgiou, H. Hadj-Salem and J.-M. Blosseville, "A local feedback control law for on-ramp metering", Transportation Research Board, vol. 1320, (1991), pp. 58-64. 
[20] M. Papageorgiou, H. Hadj-Salem and F. Middleham, “ALINEA local ramp metering:summary of field results”, Washington,DC:Transportation Research Board, (1997), pp. 90-98.

\section{Additional Information}

Competing financial interests: The authors declare no competing financial interests.

\section{Authors}

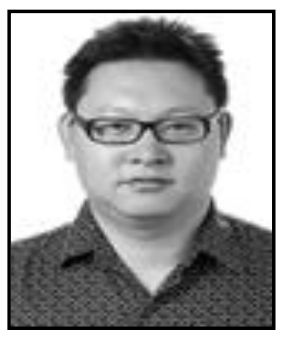

Xingju Wang, he has worked in the field of traffic engineering. He earned M.S. degree and Ph.D. degree from Gifu University in Japan from April, 2002 to June, 2008. He was a visiting scholar in the Transportation Program of the Civil, Architectural, and Environmental Engineering Department at The University of Texas at Austin from August, 2013 to May, 2014. Currently he is Professor of Traffic and Transportation Engineering at Shijiazhuang Tiedao University. He conducts research on all aspects of traffic engineering including emergency transportation, GIST and ITS.

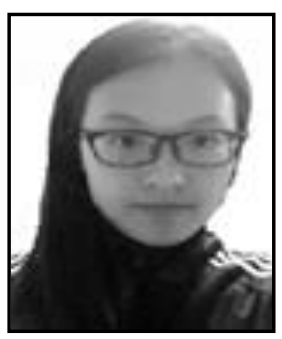

Yuehong Liu, she received her bachelor's degree in Civil Engineering in 2014 from Beijing Jiaotong University Haibin College, China. She is currently working toward the master's degree in Traffic and Transportation Engineering at Shijiazhuang Tiedao University. Her research interests include ITS, GIS-T and emergency transportation.

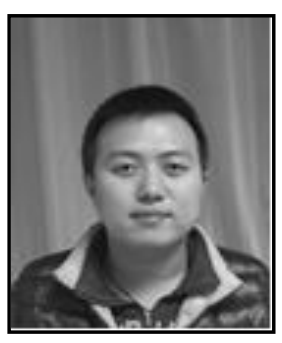

Yang Zhou, he received his bachelor's degree in Traffic Engineering in 2013 from Shijiazhuang Tiedao University, China, where he is currently working toward the master's degree. His research interests include ITS, GIS-T and emergency transportation.

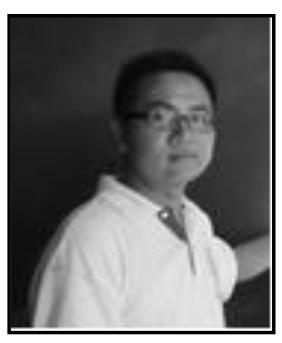

Xiaoming Xi, he received his bachelor's degree in 2009 from Tianjin Chengjian University and master's degree in 2012 from Shijiazhuang Tiedao University. His research interests include ITS, GIS-T and emergency transportation. 
International Journal of Control and Automation

Vol. 10, No. 2 (2017) 